\title{
Effects of working memory capacity on mental set due to domain knowledge
}

\author{
Travis R. RICKS \\ University of Illinois, Chicago, Illinois \\ KANDi Jo TURLEY-AmES \\ Idaho State University, Pocatello, Idaho \\ AND \\ JENNIFER WILEY \\ University of Illinois, Chicago, Illinois
}

\begin{abstract}
The present set of studies examines how working memory capacity (WMC) relates to performance on a Remote Associates Task (RAT), originally designed by Mednick (1962) as a quantifiable creative problem solving assessment. The source of fixation was manipulated across two sets of RAT items. One set was neutral with no specific fixation embedded in them, while the second set was baseball-misleading, designed so that prior knowledge of baseball would lead to an incorrect solution attempt (Wiley, 1998). WMC scores were positively related to performance on RAT items in all conditions, except one. High baseball knowledge participants' WMC scores did not relate to performance on the baseball-misleading RAT. While in general WMC may lead to better RAT performance, these results suggest that when there is a candidate solution strongly activated by prior knowledge, WMC may actually lead to too much focus on the incorrect solution and exacerbate mental sets.
\end{abstract}

Many problems we face in our everyday lives require creative problem solving. As opposed to problems that may be well-structured with obvious routes to solution, many everyday problems are ill-structured and misleading. For these problems, we need to move beyond the most obvious or salient approaches to a solution and consider a broad range of more remote possibilities or alternate representations until one fulfills our needs. The present study is concerned with how individual differences in working memory capacity (WMC) may affect performance in a particular problem solving context, the Remote Associates Task (RAT), in which many solution attempts must be generated while previous attempts need to be ignored. Since WMC has been identified as an important factor in both an individual's ability to retrieve items from long-term memory (Rosen \& Engle, 1997), as well as in the ability to resist interference during processing (Kane \& Engle, 2003; Stoltzfus, Hasher \& Zacks, 1996), the current study investigates the role WMC might play in RAT problem solving.

\section{Working Memory Capacity}

Working memory has been conceptualized as a limited capacity mental workspace in which activated memory representations are available for manipulation in a temporary buffer (Baddeley \& Hitch, 1974; Daneman \& Carpenter, 1980). Current assessments of WMC use complex span tasks in which the processing and storage components are interleaved but distinct (Conway, Kane, Bunting, Hambrick, Wilhelm, \& Engle, 2005). In complex reading span tasks, participants are asked to read sentences as a processing component and remember a list of letters as a storage component. Another measure, the operation span, involves solving elementary math problems, and remembering a list of unrelated words. When both tasks are administered, reliability of the measures can be determined, and a composite score that removes task-specific variance can be computed which typically predicts performance on a wide range of cognitive tasks, including reasoning and problem solving measures (Conway et al., 2005; Kane et al., 2004; Kyllonen, 1996; Kyllonen \& Christal, 1990).

Individual differences in WMC have been theoretically attributed to two main classes of subprocesses: differences in active maintenance or retention, and differences in attentional control or distractor blocking (Kane \& Engle, 2003). Earlier work defined WMC as the amount of activation available to the WM system (Daneman \& Carpenter, 1980; Engle, Cantor, \& Carullo, 1992), or the efficiency of encoding and retrieving information from LTM (Ericsson \& Delaney, 1998; Ericsson \& Kintsch, 1995, Rosen \& Engle, 1997). More recently, researchers have emphasized the relationship between WMC and the ability to process new information and maintain goals in the

T. R. Ricks, tricks2@uic.edu 
face of interference (Conway, et al., 2005; Rosen \& Engle, 1997; Stoltzfus, Hasher \& Zacks, 1996), or the ability to control and focus one's attention (Conway, Cowan, \& Bunting, 2001; Kane \& Engle, 2005). Kane and Engle concluded that both sets of subprocesses interact and often are jointly responsible for the effects of WMC on cognitive performance. Since RAT problem solving depends on both effective maintenance of problems in active memory, as well as attentional processes that may allow the solver to ignore previously generated solutions, in general WMC should be related to RAT performance.

\section{The Remote Associates Task}

The RAT was created by Mednick (1962) as a quantifiable creative problem solving assessment. RAT items consist of three words that appear unrelated to one another (i.e., wild, dark, and fork). Participants are then asked to generate a fourth word, which may form a meaningful phrase with each of the words. In this example, a good solution would be pitch, which would form the phrases: wild pitch, pitch dark, and pitchfork. Solving RAT items requires individuals to engage in a broad search of long-term memory to find a word that forms a meaningful phrase with all three unrelated words. It can be easy to locate words that form meaningful phrases with one or two of the words. Yet, finding a word that fits with all three words requires participants to test and reject many possible solutions. For instance horse can form the meaningful phrases wild horse and dark horse, but does not pair with fork. If horse is generated as a possible solution, individuals must abandon it and continue a search of memory to find other words that might represent a good solution to the problem. Thus, as noted above, successful RAT problem solving requires both effective search and retrieval from LTM as well as the ability to ignore previously generated solutions.

Previous studies would predict that solvers with high WMC should perform better on RAT problems as WMC has been linked to the efficient retrieval of items from long-term memory (Rosen \& Engle, 1997). Similarly, high WMC might also enhance RAT performance if it helps solvers to overcome interference from previously generated solutions by keeping their attention directed or focused on the task of finding a new solution (Kane \& Engle, 2003; Stoltzfus, Hasher, \& Zacks, 1996).

However, an interesting alternative is that high WMC or the ability to control one's attention could sometimes have negative effects if solvers are already focusing on the wrong information for a solution. This would be consistent with the effects reported by Conway, Cowan, and Bunting, (2001), who found differences in the cocktail party effect due to WMC. High WMC participants were actually less likely than low WMC participants to hear their name in an unattended channel. Thus, it appears that high WMC participants' ability to focus their attention can also limit the breadth of their awareness, and can constrain the range of information that they might consider or process. Although this ability has obvious advantages for filtering out irrelevant information in most contexts, in the case of RAT problem solving, in which a broad search for a solution is required, some negative consequences might occur.
If high WMC makes solvers focus on incorrect solution attempts, they should do worse than low WMC solvers, who may have broader activation of LTM, which would translate into better problem solving in this context.

\section{Effects of Domain Knowledge on RAT Performance}

One particular context in which this may be the case is when domain knowledge strongly activates an incorrect solution to a RAT problem. Wiley (1998) demonstrated that when domain knowledge leads to an incorrect solution attempt on RAT problems, that it establishes a mental set. To show this, Wiley created a set of RAT items specifically designed to activate a misleading baseball-related solution. Similar to the example listed earlier, these items contained a first word that was part of a familiar baseball phrase (i.e., wild, as in wild pitch). The second word in the problem also formed a meaningful phrase with this solution (i.e., dark, as in pitch dark). However, a third word did not form a pair with the obvious baseball-solution word (i.e., sense). At this point, the candidate solution pitch must be abandoned, ignored, or inhibited in some way, and generation must continue until the solution (horse) can be found.

Across three experiments, Wiley (1998) demonstrated that solvers with high baseball knowledge took longer and were less likely to solve baseball-misleading problems than low knowledge solvers. High-knowledge participants were more likely to be fixated by the solution that was suggested by their domain knowledge, and they were more likely to guess baseball-related words as solutions. In a second experiment, warning solvers not to think of baseball did not eliminate the fixation among high knowledge solvers, and if anything, the awareness that the study was about baseball made low-knowledge solvers worse on all RAT problems.

In a third experiment, to show that activation of the baseball-related solutions in memory was responsible for poorer performance of the high-knowledge participants, Wiley (1988) used a priming paradigm which exposed all solvers to the baseball-relevant solutions. After priming, the low-knowledge participants performed as poorly on misleading problems as the high-knowledge participants.

Further, in this study all participants were given a second chance to solve the problems. One half of the participants were given a break or incubation period before a second attempt at a solution, while the other half immediately attempted a solution again on all problems. The intriguing result was that the low-knowledge participants benefited more from the incubation period than the backto-back solution attempts, while the high knowledge participants showed the opposite pattern. For high-knowledge solvers, they were able to solve more previously unsolved problems when problems were presented without a break in between. An "incubation period" led to better performance for low-knowledge solvers and worse performance for the high-knowledge solvers. This result suggests that fixation or interference from prior knowledge may affect performance differently than fixation or interference produced in the context of problem solving.

In the context of the present studies, this suggests that WMC might have different effects when incorrect solu- 
tions are strongly activated by domain knowledge than when they are not. When solvers are fixated by a solution that is strongly activated due to their prior knowledge, then high WMC may actually cause them to focus more on that solution and exacerbate mental set. However, when solutions are not strongly activated by domain knowledge and are simply alternatives generated in a problem solving context, then high WMC may allow solvers to resist the interference due to previous attempts and move on. Thus, this would suggest that the effects of WMC on the solving of baseball-misleading RAT problems should vary with domain knowledge, whereas the effects of WMC on neutral problems should be generally positive.

To test this hypothesis, we compared the effects of WMC on the baseball-misleading and neutral sets of RAT problems from Wiley (1998) for participants who varied in their baseball knowledge. If WMC relates to the ability to retrieve information from memory, we would predict a positive effect of WMC on both types of RAT items. An additional prediction is that a three-way interaction should exist between WMC, domain knowledge, and RAT condition. If WMC relates to the ability to focus one's attention, then high WMC may cause high knowledge participants to focus on incorrect baseball-misleading problems (Wiley, 1998), and they may show a negative effect of WMC.

\section{EXPERIMENT 1}

\section{Method}

Participants. Participants were 61 volunteers from introductory psychology classes at Idaho State University who received course credit. Data from 3 participants were removed based on their identification as extreme outliers more than three IQRs beyond Tukey's hinges in a box plot.

Materials. An operation span test (OSPAN, Turley-Ames \& Whitfield, 2003) and Wiley's (1998) adapted version of the RAT were used in this study. The RAT contained 2 examples and 5 practice items followed by the correct answers, then 10 baseball-misleading and 10 neutral items. Two tests from Hambrick and Engle (2002) were administered to assess knowledge of baseball rules, regulations, and terminology. The fill-in-the-blank and multiple-choice tests were used as they were the two shown to be reliable in that study, and were also the most similar to the Spilich, Vesonder, Chiesi and Voss (1979) test used by Wiley (1998).
Procedure. Participants were run individually. First, participants completed the OSPAN task (Turley-Ames \& Whitfield, 2003). Each trial consisted of an operation-word sequence (i.e., 10/2 + 3 =??? TWIG). Trials were presented in sets that increased in size from two to six. During each trial, participants generated the answer to the equation and read the final word out loud. At the end of each set, participants were asked to recall the words for the set. Participants completed three trials at each set size and had seven seconds to complete each trial. Only trials in which both the solution and word were correct were given credit. The maximum WMC score was 60 points.

Following OSPAN, participants completed the RAT. In the interest of replication, presentation was identical to Wiley (1998), which used a specific order and timing to instill mental sets. The first word for each RAT item appeared in the middle of the computer screen for five seconds. The second word then appeared underneath the first for 7.5 seconds. Then, the third word appeared underneath the first and second words, and participants had 30 seconds to enter a solution. If 30 seconds elapsed without a solution being entered, the words disappeared, and participants were instructed to enter a solution. Participants had 20 seconds to respond before the next RAT item was presented. Response time was measured by computer from the presentation of the third word until the participant typed a solution and hit the enter key, or until 30 seconds had elapsed. Only the solutions listed in Wiley (1998), and slight misspellings, were accepted as correct. Participants then completed the baseball tests.

Three levels of WMC were defined by splitting WMC scores into thirds. This resulted in 22 low WMC participants whose scores ranged from 19 to 34, with an average score of 27.27 (SD 4.29), 20 medium WMC participants whose scores ranged from 34 to 41 with an average of 37.40 (2.37) and 16 high WMC participants whose scores ranged from 41 to 52 with an average score of 46.25 (SD 5.40).

Two levels of domain knowledge were defined by a median split on the combined totals for the two baseball knowledge tests. This resulted in 31 low-knowledge participants whose baseball knowledge scores ranged from 4 to 15 with an average score of 10.48 (SD 2.61), and 27 high-knowledge participants whose scores ranged from 16 to 39 with an average score of 24.48 (SD 8.0).

\section{Results}

A 2 (problem type) $\times 2$ (knowledge level) $\times 3(\mathrm{WMC})$ ANOVA was computed using number of correct solutions as the dependent measure with RAT problem type tested as a repeated measure. ${ }^{1}$ Only significant results are reported. All significant effects are followed up using Tukey's HSD with $\alpha=.05$.

Figure 1 presents the mean number correct solutions for each condition. A significant main effect was found
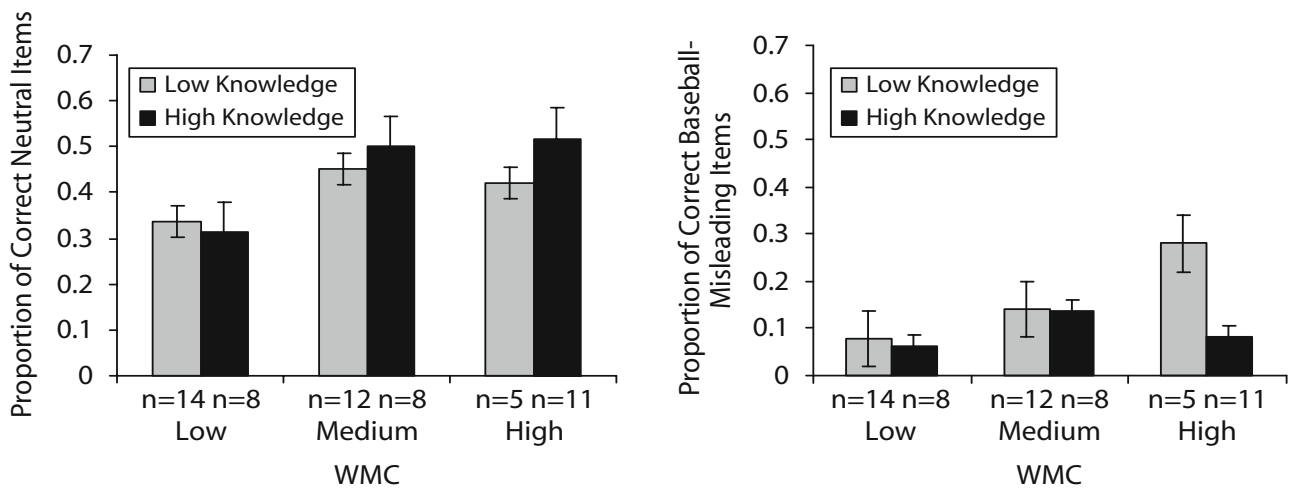

Figure 1. Proportion Correct for Neutral (left panel) and Baseball-Misleading RAT problems (right panel) by WMC and Baseball Knowledge Level in Experiment 1. Error bars represent standard errors. 
for RAT problem type $\left[F(1,52)=145.6, M S_{\mathrm{e}}=1.52\right.$, $\left.p<.01, \eta^{2}=.74\right]$. Neutral problems (left panel) were solved more often than baseball-misleading problems (right panel). A significant main effect was also found for $\operatorname{WMC}\left[F(2,52)=6.92, M S_{\mathrm{e}}=2.54, p<.01, \eta^{2}=.21\right]$, as low WMC solvers solved fewer problems than medium or high WMC solvers overall.

In replication of Wiley (1998), a significant knowledge $\times$ problem type interaction was found $[F(1,52)=$ 5.58, $\left.M S_{\mathrm{e}}=1.52, p<.02, \eta^{2}=.10\right]$. Follow up tests indicated that high knowledge participants solved fewer misleading problems than low knowledge participants, suggesting they experienced fixation on the misleading items.

Importantly, the three-way interaction was significant $\left[F(1,52)=3.30, M S_{\mathrm{e}}=1.52, p<.05, \eta^{2}=.11\right]$. The nature of this interaction is best seen in Figure 1. This interaction was explored by looking at the two types of RAT problems separately. The two-way interaction was not significant for neutral problems. Only the main effect for WMC was significant as low WMC solvers solved fewer neutral problems than medium or high WMC solvers $\left[F(1,52)=4.25, M S_{\mathrm{e}}=3.26, p<.02, \eta^{2}=.14\right]$.

On misleading problems, however, both main effects and the interaction were significant. Performance improved with WMC $\left[F(1,52)=6.74, M S_{\mathrm{e}}=.80, p<\right.$ $.01, \eta^{2}=.21$, low WMC performed worse than medium or high WMC] and decreased with domain knowledge $\left[F(1,52)=8.60, M S_{\mathrm{e}}=.80, p<.01, \eta^{2}=.14\right]$. Follow-up tests indicated that the significant interaction $[F(1,52)=$ $\left.5.67, M S_{\mathrm{e}}=.80, p<.01, \eta^{2}=.18\right]$ was due to the low knowledge individuals with the highest level of WMC outperforming all other low knowledge individuals, and performing significantly better than high WMC, high knowledge individuals.

Solution time data were analyzed using the same ANOVA approach, but no significant effects or interactions were found.

\section{Discussion}

Experiment 1 did find a positive overall effect of WMC on RAT problem solving, which is consistent with previous work by Rosen and Engle (1997) who linked WMC to efficient retrieval from LTM. In a replication of Wiley (1998), the high knowledge participants in this study also experienced more difficulty on the baseball-misleading problems than did the low-knowledge participants. Interestingly, in the baseball-misleading condition, the relation of WMC to RAT performance differed between high and low knowledge solvers. For low knowledge participants, there was again a positive relation between WMC and performance. However, for high knowledge individuals, high WMC scores did not lead to better performance.

The significant three-way interaction between WMC, domain knowledge, and problem type offers support for the prediction that when strongly activated prior knowledge fixates a participant on a solution attempt, high WMC may have a negative influence on problem solving.

While this study suggests an interesting relationship between WMC and RAT problem solving, the small sample size and the low rate of solution on the baseballmisleading items is a concern. One possible reason for the low level of performance on the baseball-related items is that participants in this study may have been aware that the study was about baseball before they arrived. This research was performed on the small campus of Idaho State University, and it was noted by the researchers that numerous participants in this experiment asked the research assistants if they were about to participate in "the baseball study." This suggests that many of the participants may have been primed to think about baseball during the experiment. Thus, these findings may be similar to the second experiment of Wiley (1998) in which participants were warned not to think about baseball related solution, which ironically caused fixation among baseball novices. In Experiment 2, we replicate this study at a larger campus where it is less likely that the topic of the experiment would be known.

\section{EXPERIMENT 2}

This experiment attempts to replicate the results of Experiment 1 with a larger sample, at an institution where it is easier to conceal the baseball-related content of the experiment.

\section{Method}

Participants. Participants were 113 students in the Introductory Psychology Subject Pool at the University of Illinois at Chicago who received course credit for their participation. Ten participants were removed for having no correct answers on the Baseball Knowledge Questionnaire (in which case zero scores could not be distinguished from a lack of effort). Data from 2 participants were removed as extreme outliers using Mahalanobis distances. Baseball knowledge scores ranged from 1 to 40 , with an average score of 13.21 ( $S D$ 11.89). WMC scores ranged from 11.5 to 39.5 with an average score of 25.97 ( $S D$ 6.25).

Materials and Procedure. There were several differences from the materials and procedures used for the first experiment. Participants were administered both the OSPAN and RSPAN according to the guidelines posited by Conway and colleagues (2005) in order to derive a composite "proportion correct" score that removed taskspecific variance. An example of an OSPAN trial on this task is $10 / 2+3=2$ TWIG, in which the participant verifies whether the equation is correct, and then reads the final word out loud. An example of an RSPAN trial is "Andy crossed the yellow heaven. J" in which the participant verifies if the sentence makes sense and then reads the letter out loud. Trials were presented in sets, and participants were asked to recall all words or letters at the end of each set. Set sizes for both span tasks were randomized between sizes of two and five.

Administration of the WMC task was performed in a session independent of the RAT. WMC tasks were run individually. RAT tasks were run in groups ranging from 1 to 10 . Following the scoring procedure listed in Wiley (1998), alternate solutions that were rated as being as acceptable as the intended solutions, as well as the intended solutions, and slight misspellings, were accepted as correct. Finally, participants were administered the same baseball knowledge questionnaire that was used in Wiley (1998).

\section{Results}

Regression analyses were conducted to evaluate the main and interactive effects of baseball knowledge, WMC, and condition on RAT performance. RSPAN and 

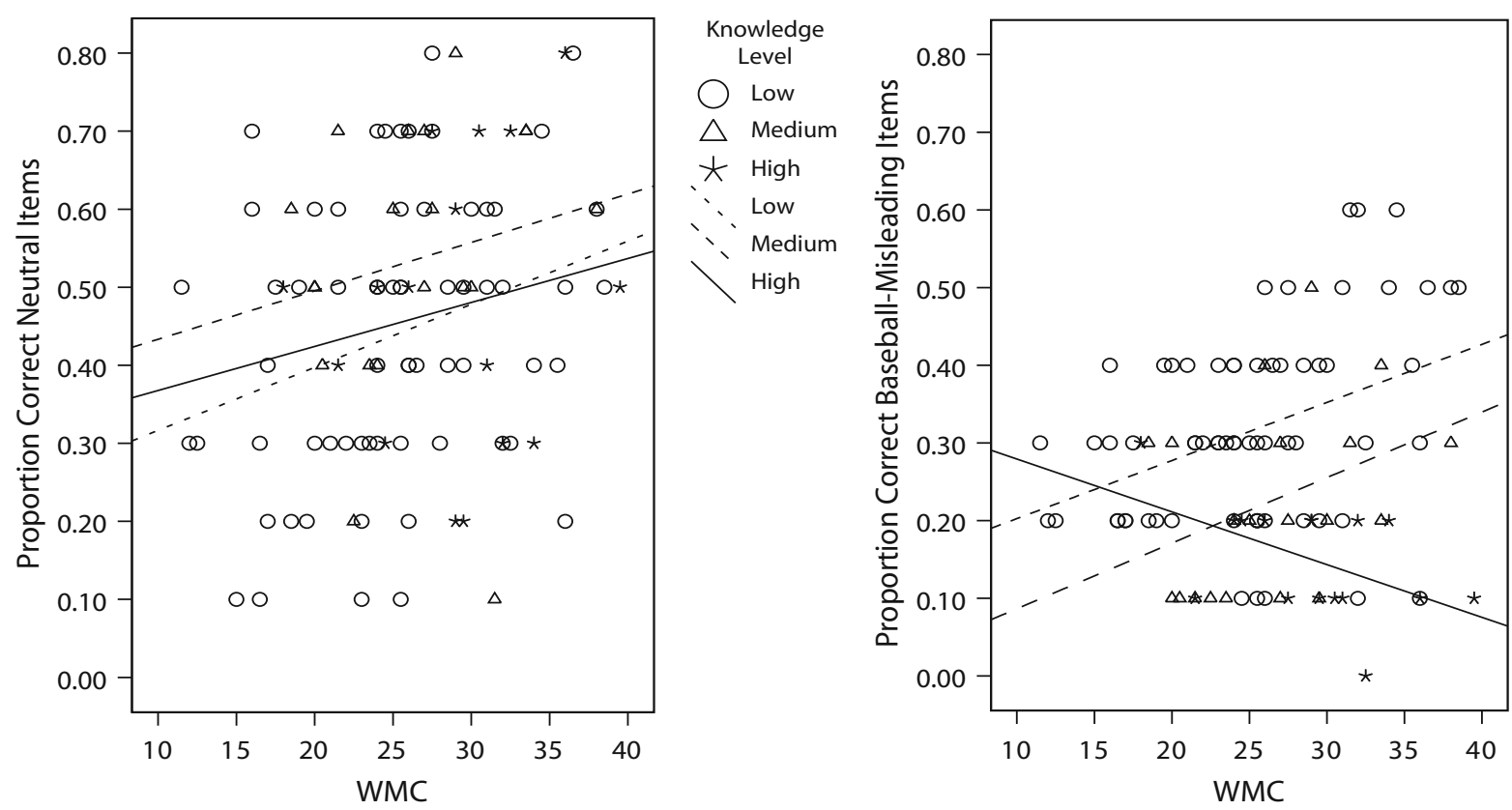

Figure 2. Proportion correct solutions for Neutral (left panel) and Baseball-Misleading RAT problems (right panel) by WMC and Baseball Knowledge Level in Experiment 2. Markers for low-knowledge participants are circles, triangles for medium knowledge and stars for high knowledge. The dotted regression line with the smallest dashes is for lowknowledge, the medium dashes for medium-knowledge, and the solid line is for the high-knowledge participants.

OSPAN scores were correlated at .67 in our sample, demonstrating the reliability of these measures. For use as predictor variables, the working memory span composite score and baseball knowledge test scores were converted to Z-scores.

The relation of working memory capacity to RAT performance as a function of RAT items and baseball knowledge is presented in Figure 2. To ease interpretation of the regression results, graphs displaying the relation of WMC to RAT performance as a function of RAT condition and baseball knowledge are presented. For illustration purposes, baseball knowledge is graphed at 3 levels: 15 items correct or less, which represents low knowledge, between 15 and 30 items correct, which represents moderately high baseball knowledge, and 30 or more items correct, which corresponds to the highest level of baseball knowledge in the meta-analysis of the three Wiley (1998) experiments.

When neutral RAT performance was examined alone in a regression entering WMC, baseball knowledge score, and the interaction, there was a positive effect of WMC ( $\beta=.21, t=2.15, p<.03)$ and a marginal positive effect for baseball knowledge $(\beta=.17, t=1.66, p<.10)$, with a nonsignificant interaction $(\beta=-.12, t=1.1, p<$ .26).

When baseball RAT performance was examined alone, there was again positive effect of WMC $(\beta=.26, t=$ $2.94, p<.01)$, but now a significant negative effect for baseball knowledge $(\beta=-.47, t=5.36, p<.001)$, and a significant interaction $(\beta=-.22, t=2.25, p<.01)$.

To test for the three-way interaction observed in Experiment 1 , a hierarchical analysis that examined performance on baseball-misleading RAT problem while controlling for performance on neutral problems was carried out in three steps. The number of neutral RAT items solved, baseball knowledge, and WMC were entered in the first step to evaluate the main effect of each predictor variable on performance of the baseball-misleading RAT items. Cross-product terms representing baseball knowledge $\times$ WMC, baseball knowledge $\times$ neutral RAT performance, and $\mathrm{WMC} \times$ neutral RAT performance two-way interactions were entered in the next step. A cross-product of baseball knowledge $\times$ WMC $\times$ neutral RAT performance, representing the three-way interaction, was entered in the third step.

Table 1 summarizes the outcome of the hierarchical regression analysis. WMC, baseball knowledge, and neutral RAT performance were all significant, unique predictors

Table 1

Hierarchical Regression Analysis for RAT Performance in Experiment 2

\begin{tabular}{lccrcc}
\hline \multicolumn{1}{c}{ Variable } & Inc $R^{2}$ & $F$ & $B$ & $t$ & $s r^{2}$ \\
\hline Step 1 & .33 & $15.9^{* *}$ & & & \\
WMC & & & .030 & $2.55^{* *}$ & .045 \\
BK & & & -.074 & $6.34^{* *}$ & .278 \\
$\quad$ Condition & .084 & $4.48^{* *}$ & .189 & $2.96^{* *}$ & .061 \\
Step 2 & & & -.179 & $2.47^{*}$ & .038 \\
$\quad$ BK $\times$ condition & & & .151 & $2.23^{*}$ & .031 \\
WMC $\times$ condition & & & -.029 & $2.43^{*}$ & .036 \\
WMC $\times$ BK & .027 & $4.56^{*}$ & & & \\
Step 3 & & & -.173 & $2.14^{*}$ & .028 \\
$\quad$ WMC $\times$ BK $\times$ condition & & &
\end{tabular}

Note-Inc $R^{2}$, increment in variance accounted for; $B$, unstandardized regression coefficient; $s r^{2}$, squared semipartial correlation; WMC, working memory capacity; BK, baseball knowledge. ${ }^{*} p<.05 .{ }^{* *} p<.01$. 
of correct solutions. Higher levels of WMC and neutral RAT performance were associated with superior performance on the baseball-misleading RAT items. Higher baseball knowledge was related to poorer performance.

In addition, all of the two-way interactions were significant. This included the two-way interaction between baseball knowledge and RAT condition (measured by the baseball knowledge $\times$ neutral RAT performance cross-product), which replicates the findings of Wiley (1998) and shows that participants are less likely to solve baseball-misleading problems, but not neutral problems, with increasing baseball knowledge. The working memory by condition interaction (i.e., WMC $\times$ neutral RAT performance) was due to RAT performance in the neutral condition increasing with WMC more so than in the baseball-misleading condition. The WMC by Baseball Knowledge interaction was due to RAT performance increasing with WMC for low-knowledge participants, but decreasing with WMC for high-knowledge participants.

However, these effects were all subsumed by the significant three-way interaction. The nature of the three-way interaction can be seen by comparing the two panels in Figure 2. As shown in the left panel, participants at all levels of domain knowledge showed a positive relation between WMC and correct solutions in the neutral condition. However, as shown in the right panel, the highest knowledge individuals showed a negative relation between WMC and correct solutions in the misleading condition (represented by the solid regression line), whereas participants at lower levels of knowledge showed a positive relationship with WMC (represented by the dashed regression lines). This significant interaction again suggests that WMC can impair performance on the baseball-misleading items for high knowledge individuals. (Adjusted means for baseball performance after controlling for neutral performance followed the same pattern as shown in the right panel of Figure 2.)

Solution time data were analyzed using the same regression approach, but no significant effects or interactions were found.

\section{GENERAL DISCUSSION}

The present results provide support that both active maintenance and controlled attention processes lead to effects of WMC on problem solving. Both studies found evidence that WMC was positively related to RAT problem solving ability on neutral RAT problems. This may be explained by the fact that RAT problems require the generation of remote solution candidates as well as the ability to disregard previous solutions that were generated in the context of the problem. This result is, thus, consistent with Rosen and Engle (1997) who showed that WMC is related to efficient retrieval from LTM, as well as findings that WMC is related to the ability to resist interference and inhibit irrelevant information (Stoltzfus, Hasher \& Zacks, 1996). Although present results cannot differentiate whether effects were obtained because of differences in WMC per se versus effects of close correlates such as $g$ or STM capacity, the positive effects can be explained by any "improved retention" account of WMC that involves more effective STM maintenance, or better LTM storage and retrieval processes.

While "improved retention" approaches to WMC can explain the overall positive effect of WMC on RAT performance, only the controlled attention perspective can explain why negative effects of WMC were found when incorrect solutions were strongly activated by prior knowledge. According to this approach (Kane \& Engle, 2003), high WMC enables solvers to focus their attention. But, in the case of high knowledge participants, that attention was being focused on an incorrect solution. The findings that WMC had a negative effect on high knowledge participants' ability to solve baseball-misleading items is consistent with the hypothesis that WMC can under certain conditions produce too much focus (Conway, Cowan \& Bunting, 2001). When a solution was highly activated by prior knowledge, then the effects of WMC exacerbated mental set on incorrect solutions. In both studies the low WMC participants with high levels of baseball knowledge were not as affected by the baseball-misleading material as high WMC participants.

In terms of a contribution to the WMC literature, this finding lends support to the controlled attention perspective, which argues that one means by which working memory capacity affects cognitive performance is through the executive ability to direct or focus one's attention (Kane \& Engle, 2003). Sometimes this control allows high WMC performers to overcome interference, as has been shown in dichotic listening and Stroop tasks. However, the present study shows that the effects of attentional focus are not always positive and can make participants focus on stimuli that does not facilitate their performance. In the case of creative problem solving tasks in which a broad search for solution is required, this ability may be detrimental. We argue that high WMC participants' ability to focus their attention constrained the range of information that was considered during problem solving. This ability has obvious advantages for filtering out irrelevant information when it is clear what information is relevant. However, when a strong associate that is being activated from prior knowledge seems relevant, but is not; some negative consequences may occur. In particular, high WMC solvers in this study may have filtered out or failed to consider remote associates, which may have seemed irrelevant, but, in fact, these associates were the candidates that would have actually led to a solution.

This argument would be more convincing if we had evidence of fixation by incorrect baseball solutions in these studies. In Wiley (1998), a higher rate of baseball-related incorrect guesses among high-knowledge participants supported the fixation explanation. In the present studies, solvers were not asked to report the possible solutions that they attempted, so we cannot say with certainty that the present effects are due to fixation on incorrect solutions. Evidence of fixation on baseball-related solutions from think-aloud protocols, or priming experiments that test for activation for baseball-related solutions, are important future directions for this work. Also, while we suspect the low rate of success on baseball items in the first study was 
due to a general awareness that the experiment was about baseball in that sample, it would be interesting to see if a warning not to think about baseball might interact with working memory capacity.

The present results show the effects of WMC and domain knowledge on problem solving are not straightforward. WMC and domain knowledge may predict different aspects of the problem solving process (Ash \& Wiley, 2006) and, in some contexts, may have interactive effects. WMC may influence the number of solution attempts that can be generated or retrieved from memory, as well as whether a solver is able to move past initial attempts to solve the problem. Domain knowledge may help solvers to generate solutions in many cases, but in other cases domain knowledge may constrain solution generation and cause mental sets that can interact with WMC. An interesting result of the present research is the additional evidence it provides that there are important differences in problem solving when fixation comes from prior knowledge (i.e., Wiley, 1998) versus when it is instilled by the context of the problem or prior solution attempts (i.e., Luchins \& Luchins, 1959). The data presented here suggest that WMC seems to help solvers escape the interference from the latter, and not the former. Thus, these results also may help advance a theory of effective problem solving that considers how the source of fixation in problem solving may interact with individual differences in determining whether successful solutions can be achieved.

\section{AUTHOR NOTE}

The research reported here served as part of the first author's thesis, submitted to the Department of Psychology, Idaho State University, in partial fulfillment of requirements for the MS degree. We thank Gary Anderson for programming the software for the first study and Melinda $\mathrm{S}$. Jensen for her comments on a draft of the manuscript. Portions of this research were supported by the National Science Foundation under Grant 0347887 to the third author. Any opinions, findings, and conclusions or recommendations expressed in this material are those of the authors and do not necessarily reflect the views of the National Science Foundation. Correspondence relating to this article may be sent to T. R. Ricks, Department of Psychology, 1007 W. Harrison St. (M/C 285), Chicago, IL 60607 (e-mail: tricks2@uic.edu).

\section{REFERENCES}

Ash, I. K., \& WiLEy, J. (2006). The nature of structuring in insight: An individual-differences approach. Psychonomic Bulletin \& Review, 13, 66-73.

BADDELEY, A. D., \& Hitch, G. J. (1974). Working memory. In G. H. Bower (Ed.), The psychology of learning and motivation (Vol. 8, pp. 47-89). New York: Academic Press.

Conway, A. R. A., Cowan, N. C., \& Bunting, M. F. (2001). The cocktail party phenomenon revisited: The importance of working memory capacity. Psychonomic Bulletin \& Review, 8, 331-335.

Conway, A. R. A., Kane, M. J., Bunting, M. F., Hambrick, D. Z., Wilhelm, O., \& ENGLE, R. (2005). Working memory span tasks: A methodological review and users guide. Psychonomic Bulletin \& Review, 2, 769-786.

Daneman, M., \& CARPEnter, P. A. (1980). Individual differences in working memory and reading. Journal of Verbal Learning \& Verbal Behavior, 19, 450-466.

Engle, R. W., Cantor, J., \& Carullo, J. J. (1992). Individual differences in working memory comprehension: A test of four hypotheses. Journal of Experimental Psychology: Learning, Memory, \& Cognition, 9, 972-992.

Ericsson, K. A., \& Delaney, P. F. (1998). Working memory and expert performance. In R. H. Logie \& K. J. Gilhooly (Eds.), Working memory and thinking (pp. 93-114). Hove, U.K.: Psychology Press.

ERICSSON, K. A., \& KinTsCH, W. (1995). Long-term working memory. Psychological Review, 102, 211-245.

Green, S. B. (1991). How many subjects does it take to do a regression analysis? Multivariate Behavioral Research, 26, 499-510.

Hambrick, D. Z., \& Engle, R. W. (2002). Effects of domain knowledge, working memory capacity, and age on cognitive performance: An investigation of the knowledge is power hypothesis. Cognitive Psychology, 44, 339-387.

KANE, M. J., \& ENGLE, R. W. (2003). Working-memory capacity and the control of attention: The contributions of goal neglect, response competition, and task set to Stroop interference. Journal of Experimental Psychology: General, 132, 47-70.

Kane, M. J., Hambrick, D. Z., Tuholski, S. W., Wilhelm, O., Payne, T. W., \& ENGLE, R. W. (2004). The generality of working memory capacity: A latent variable approach to verbal and visuospatial memory span and reasoning. Journal of Experimental Psychology: General, 133, 189-217.

KYLLONEN, P. C. (1996). Is working memory capacity Spearman's G? In I. Dennis \& P. Tapfield (Eds.), Human abilities: Their nature and measurement (pp. 49-75). Mahwah, NJ: Erlbaum.

Kyllonen, P. C., \& Christal, R. E. (1990). Reasoning ability (is more than) working memory capacity?! Intelligence, 14, 389-433.

LUCHINs, A. S., \& LUCHINs, E. H. (1959). Rigidity of behavior: A variational approach to the effect of Einstellung. Eugene: University of Oregon Press.

Mednick, S. (1962). The associative basis of the creative problem solving process. Psychological Review, 69, 200-232.

Rosen, V. M., \& ENGLE, R. W. (1997). The role of working memory capacity in retrieval. Journal of Experimental Psychology: General, 126, 211-227.

Spilich, G. J., Vesonder, G. T., Chiesi, H. L., \& Voss, J. F. (1979). Text processing of domain-related information for individuals with high- and low-domain knowledge. Journal of Verbal Learning \& Verbal Behavior, 18, 275-290.

Stoltzfus, E. R., Hasher, L., \& Zacks, R. T. (1996). Working memory and aging: Current status of the inhibitory view. New York: Oxford University Press.

Turley-AmEs, K. J., \& Whitfield, M. M. (2003). Strategy training and working memory task performance. Journal of Memory \& Language, 49, 446-468.

TURner, M. L., \& ENGLE, R. W. (1989). Is working memory capacity task dependent? Journal of Memory \& Language, 28, 127-154.

WiLEY, J. (1998). Expertise as mental set: The effects of domain knowledge in creative problem solving. Memory \& Cognition, 26, 716730 .

\section{NOTE}

1. ANOVA was computed instead of multiple regression due to the small ratio of cases to independent variables in this sample (Green, 1991), as well as a skewed distribution for baseball knowledge with more participants at lower levels of knowledge. Despite uneven cell sizes, the assumption of homogeneity of variance was not violated.

(Manuscript received July 8, 2006; revision accepted for publication September 6, 2006.) 